\title{
Three-Dimensional Trapping and Dynamic Axial Manipulation with Frequency-Tuned Spiraling Acoustical Tweezers: A Theoretical Study
}

\author{
Zhixiong Gong ${ }^{1}{ }^{1}$ and Michael Baudoin ${ }^{1,2, *}$ \\ ${ }^{1}$ Université Lille, CNRS, Centrale Lille, Université Polytechnique Hauts-de-France, UMR 8520_IEMN-Institut \\ d'Électronique de Microélectronique et de Nanotechnologie, Lille F-59000, France \\ ${ }^{2}$ Institut Universitaire de France, 1 rue Descartes, Paris 75005, France
}

(Received 2 April 2021; revised 8 July 2021; accepted 30 July 2021; published 20 August 2021)

\begin{abstract}
Holographic acoustical tweezers based on Archimedes-Fermat spiraling interdigitated transducers (SIDTs) are a versatile tool for the selective manipulation of microparticles [Baudoin et al., Sci. Adv. 5, eaav1967 (2019)] and cells [Baudoin et al., Nat. Commun., 11, 4244 (2020)] in a standard microfluidic environment. These binary active holograms produce some focused helical wave, with the ability to trap particles at the vortex core. Yet, all the studies conducted with SIDTs have so far been restricted to two-dimensional (2D) manipulation only. Here we show through a theoretical study that (i) a 3D radiation trap for microparticles and cells can be obtained with spiraling tweezers with sufficiently large aperture and that (ii) the particles can be displaced axially by simply tuning the driving frequency, without any motion of the transducer. This work opens perspectives for 3D cells and microparticles manipulation with single-beam acoustical tweezers.
\end{abstract}

DOI: 10.1103/PhysRevApplied.16.024034

\section{INTRODUCTION}

The idea of manipulating objects with the acoustic radiation force was instilled by the early theoretical works of Rayleigh [1,2], Langevin [3,4], and Brillouin [5,6] on the acoustic radiation pressure and the calculations of the force applied by an acoustic field on a spherical particle [7-9]. Experimentally, some early demonstrations of particles trapping at the nodes and antinodes of standing waves depending on their properties were reported at the beginning of the twentieth century [10-12]. Since then, this principle has been refined, e.g., by combining highfrequency surface acoustic wave orthogonal transducers and microfluidics techniques to manipulate microparticles and cells in two dimensions in a microchannel $[13,14]$, or by using static [15] or reconfigurable holograms [16-20] to pattern or manipulate many particles simultaneously in two and three dimensions. In acoustics, the term "acoustical tweezers" was introduced by $\mathrm{Wu}$ [21] in analogy with optical tweezers. But in this work, like in all the aforementioned works, the acoustical tweezers lack two essential features of their optical counterpart [22]: (i) the selectivity, i.e., the ability to select, trap, and manipulate a single object without affecting other neighboring objects and (ii) three-dimensional trapping capability with a single beam, i.e., the ability to produce a three-dimensional

\footnotetext{
*Corresponding author. michael.baudoin@univ-lille.fr; http:// films-lab.univ-lille1.fr/michael
}

(3D) trap from a beam produced from only one side of the manipulation chamber.

The selectivity requires that the spatial localization of the acoustic energy affects only the targeted object [23]. This localization can be achieved with focused beams, as investigated in optics [22] and later in acoustics [24]. But in acoustics, many particles of interest (in particular, those with positive contrast factors) are expelled, not trapped, at the center of a focused beam, or can be trapped only laterally at very specific frequencies close to the particle resonances [25]. To trap this type of particle, one possibility is to use acoustical vortices [23] (Bessel beams of topological order larger than or equal to one), some helical wavefields spinning around a phase singularity, investigated in acoustics by Hefner and Marston [26]. At first, the potential of cylindrical acoustical vortices to trap particles in two dimensions has been investigated both theoretically [27] and experimentally [28]. Such wavefields can be produced with an array of bulk [28-30] or surface acoustic wave transducers [31-33], passive holograms based on diffraction gratings [34-36], phase plates [37] and metameterials [38], or finally active holograms based on spiraling interdigitated transducers (SIDTs) [39]. Transducer arrays have the advantage of producing reconfigurable acoustic fields, which can be controlled electronically to move the object. As a counterpart, these systems require a complex array of transducers, which can be complicated to manufacture at ultrasonic high frequencies due to cross-coupling and high-end costly electronics, which both severely limit the 
applicability of this method to trap microscopic particles. Passive holograms based on phased lens and diffraction gratings turn the signal produced by a single plane-wave transducer into the desired wavefield. The advantage is the simplicity of the system. But, due to the diffraction limit, the phased lens or diffraction grating engineering precision $d$ should not be larger than half of the wavelength $d \leq \lambda / 2$ [15], which severely limits the frequency range that can be achieved. The SIDTs on the other hand rely on spiraling electrodes patterned at the surface of a transparent piezoelectric substrate with classic photolithography techniques. Hence, they are flat, transparent, easily integrable in a microscope [39], and should enable vortex synthesis up to gigahertz frequency [40].

With tweezers based on cylindrical vortices, (i) the selectivity remains limited [23] since the beam is only focused laterally, leading to spurious secondary rings whose intensity decreases as $1 / r^{2}$; (ii) particles can only be pushed or pulled [41-44] but not trapped in the axial direction, owing to the invariance of the intensity profile in this direction. Three-dimensional tweezers require that the particle is subjected to a restoring acoustic radiation force from all directions at the same time. These two problems (reduced selectivity and 3D trapping inability) can be addressed by using focused acoustical vortices or spherical vortices, as demonstrated theoretically [45] and later experimentally [46] by Baresch et al. in water. Levitation with vortex beams was also demonstrated in air by Marzo et al. [47], who also investigated the possibility offered by other types of beam such as twin and bottle beams. Applicability of these systems for in-vivo manipulation of millimeter-size objects in the urinary bladders of live pigs has recently been demonstrated [48], as well as the in-vivo collection of microbubbles within mouse capillaries [49].

Nevertheless these systems based on transducer arrays suffer the same limitations as those previously mentioned in the 2D case for micrometric objects in a standard microscopy environment. Jimenez et al. [50] and Baudoin et al. [51,52] demonstrated that focused vortices can be produced with passive Fresnel spiral zone plates and active SIDTs with Archimedes-Fermat geometry, respectively. In particular, Baudoin et al. [51,52] were able to synthesize high-frequency acoustical vortices and demonstrate selective trapping and displacement of microparticles [51] and cells [52] in a standard microscopy environment, with forces several orders larger than their optical counterparts [52]. Nevertheless, the 3D trapping capabilities of these SIDT-based tweezers were not investigated since the experiments were conducted in narrow microfluidic chambers with reduced axial dimension. Also, one difficulty with these integrated systems is that the transducers cannot be displaced axially to keep contact with the microfluidic chamber, hence preventing axial manipulation of the particles. Recently, it was shown in airborne experiments [53] that the focal point of a focused vortex synthesized with a spiraling structure can be moved axially by tuning the activation frequency, while also leading to some slight distortion of the acoustical vortex.

In this paper, we theoretically show that (i) a 3D radiation trap for microparticles and cells can be produced with single beam SIDT tweezers and that (ii) the 3D trap is maintained and displaced axially when tuning the driving frequency.

\section{METHOD: ANGULAR SPECTRUM BASED CALCULATION OF THE 3D ACOUSTIC RADIATION FORCE}

The 3D radiation force applied on a microparticle by an acoustic field produced by Archimedes-Fermat spiraling transducers is calculated with an in-house code [52] based on the angular spectrum method (ASM) originally introduced by Sapozhnikov and Bailey [54]. An outline of this method and a comparison to other existing formulas $[27,55]$ of the $3 \mathrm{D}$ acoustic radiation force applied on a particle of arbitrary size is discussed in Ref. [56].

The flowchart for using ASM to compute the wave propagation is given and discussed in detail in Appendix A. Here, we briefly review the ASM-based radiation force formulas. The incident field in a source plane is decomposed into a sum of plane waves using the angular spectrum decomposition (the 2D spatial Fourier transform of the incident field). Then the known decomposition of plane waves into spherical harmonics and the Legendre addition theorem are used to express the incident field in the spherical harmonics basis weighted by the ASM-based beam shape coefficients $H_{n m}$ :

$$
p_{i}=\frac{1}{\pi} \sum_{n=0}^{\infty} \sum_{m=-n}^{n} i^{n} H_{n m} j_{n}(k r) Y_{n}^{m}(\theta, \varphi)
$$

with

$$
H_{n m}=\iint_{k_{x}^{2}+k_{y}^{2} \leq k^{2}} S\left(k_{x}, k_{y}\right) Y_{n}^{m *}\left(\theta_{k}, \varphi_{k}\right) d k_{x} d k_{y} .
$$

Here $S\left(k_{x}, k_{y}\right)$ is the so-called angular spectrum, $j_{n}(k r)$ and $Y_{n}^{m}$ are respectively the spherical Bessel function of the first kind and the spherical harmonics, $k_{x}$ and $k_{y}$ are the wavevector (k) components in the $x$ and $y$ directions with the relations to angles $\cos \theta_{k}=\left[1-\left(k_{x}^{2}+k_{y}^{2}\right) / k^{2}\right]^{1 / 2}$ and $\varphi_{k}=\arctan \left(k_{y} / k_{x}\right)$.

The scattering problem is solved by using known results for planed waves [54,57], leading to

$$
p_{s}=\frac{1}{\pi} \sum_{n=0}^{\infty} \sum_{m=-n}^{n} i^{n} H_{n m} A_{n}^{m} h_{n}^{(1)}(k r) Y_{n}^{m}(\theta, \varphi),
$$

where $h_{n}^{(1)}$ is the spherical Hankel function of the first kind and the $A_{n}^{m}$ are the partial wave coefficients, which 
depend on the acoustic materials and geometric shape of the scatterer, and the boundary condition between the fluid and the particle. Finally, the force is obtained by integrating Brillouin's tensor [5] on a still surface in the far field
$[58,59]$, leading to the equations of the $3 \mathrm{D}$ radiation force applied on a sphere in an arbitrary acoustic field obtained by Sapozhnikov and Bailey [54] and slightly recast here under the form [56]

$$
\begin{aligned}
& F_{x}=\frac{1}{4 \pi^{2} \rho_{0} k^{2} c_{0}^{2}} \operatorname{Re}\left\{\sum_{n=0}^{\infty} \sum_{m=-n}^{n} C_{n}\left(-b_{n+1}^{-m} H_{n m} H_{n+1, m-1}^{*}+b_{n+1}^{m} H_{n m} H_{n+1, m+1}^{*}\right)\right\} \\
& F_{y}=\frac{1}{4 \pi^{2} \rho_{0} k^{2} c_{0}^{2}} \operatorname{Im}\left\{\sum_{n=0}^{\infty} \sum_{m=-n}^{n} C_{n} b_{n+1}^{m}\left(H_{n,-m} H_{n+1,-m-1}^{*}+H_{n m} H_{n+1, m+1}^{*}\right)\right\} \\
& F_{z}=-\frac{1}{2 \pi^{2} \rho_{0} k^{2} c_{0}^{2}} \operatorname{Re}\left\{\sum_{n=0}^{\infty} \sum_{m=-n}^{n} C_{n} c_{n+1}^{m} H_{n m} H_{n+1, m}^{*}\right\}
\end{aligned}
$$

where $\quad C_{n}=A_{n}+2 A_{n} A_{n+1}^{*}+A_{n+1}^{*}$,

$b_{n}^{m}=\sqrt{[(n+m)(n+m+1)] /[(2 n-1)(2 n+1)]}$,

$c_{n}^{m}=$ $\sqrt{[(n+m)(n-m)] /[(2 n-1)(2 n+1)]}$, and $\rho_{0}$ and $c_{0}$ are the density and sound speed in the outside fluid medium. Note that here the partial wave coefficients $A_{n}^{m}$ reduce to $A_{n}$ owing to the spherical shape of the particle.

\section{THREE-DIMENSIONAL TRAPPING WITH SIDT-BASED TWEEZERS}

\section{A. HAT design and acoustic field}

Most particles of interest for microfluidic applications (e.g., solid microparticles) and biological applications (e.g., cells in water) are stiffer and denser than the surrounding medium and cannot be trapped in three dimensions with a one-sided focused beam [25].
Three-dimensional selective trapping of such particles requires specific wavefields such as focused acoustical vortices [23]. Here we investigate the trapping capabilities of focused one-sided vortex beams resembling the fields synthesized by the SIDTs used in Refs. [51,52,57]. The focused vortex is produced by two Archimedes-Fermat spiraling electrodes of inverse polarity obtained by discretizing the intersection of an acoustical vortex with a plane on two phase levels. The exact transducer geometry (a)

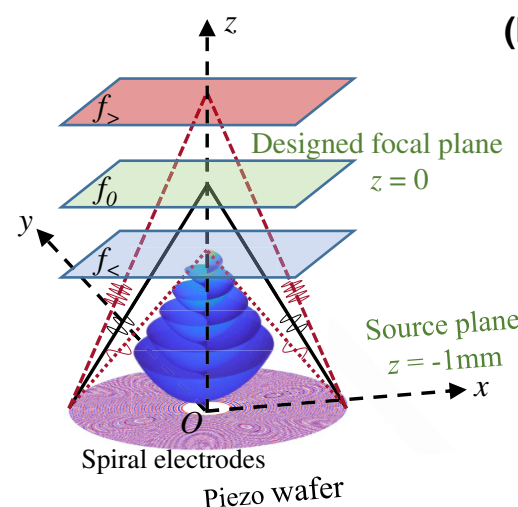

(b)

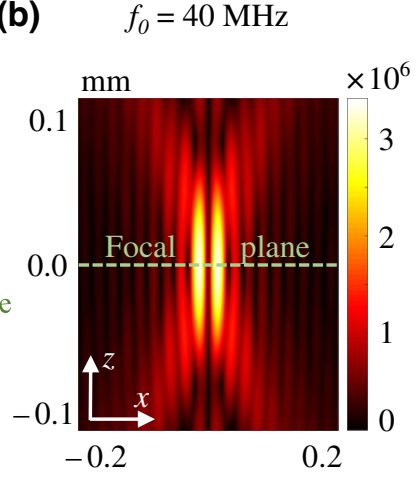

(c)
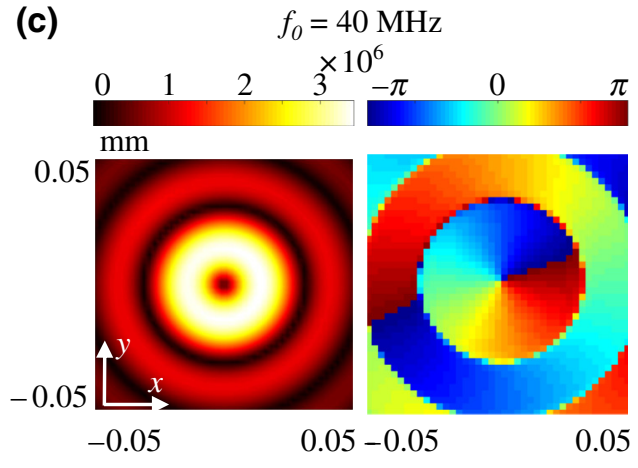

FIG. 1. Field produced by a one-sided holographic acoustical tweezer (HAT) designed to synthesize a focused vortex configuration. (a) The HAT is made of two intertwined spiraling electrodes of inverse polarity whose equations are given in Appendix B. It is designed to synthesize a focused vortex at the original driving frequency $f_{0}=40 \mathrm{MHz}$ (wavelength $\lambda=37.5 \mu \mathrm{m}$ ) and focal plane at heights $z=0,1 \mathrm{~mm}$ away from the source plane $z=-1 \mathrm{~mm}$. The origin in the lateral plane is located at point $O$ that is the center of the spiraling electrodes. The focal plane along the propagation direction can be changed by changing the actuation frequency. When the excitation frequency $f_{>}$is higher than the designed frequency $f_{0}$, i.e., $f_{>}>f_{0}$, the focal plane moves away from the source plane, while when $f_{<}<f_{0}$, the focal plane moves closer to the source plane. (b) Pressure amplitude in the propagation plane $(x, y=0, z)$ at actuation frequency $f_{0}$. (c) Pressure amplitude and phase in the focal plane $(x, y, z=0)$ at actuation frequency $f_{0}$. 
and source field used in the present simulations are given in Appendix B and the resulting acoustic field is represented in Fig. 1. The transducer is designed to produce a focused vortex with an aperture of $65^{\circ}$ and a focal plane located $1 \mathrm{~mm}$ away from the transducer when actuated at the original frequency $f_{0}=40 \mathrm{MHz}$. The pressure amplitude in the propagation plane $(x, y=0, z)$ and the focal plane $(x, y, z=0)$ are represented in Figs. 1(b) and 1(c), respectively.

We now investigate (i) the ability of such an acoustic field to trap a particle or a cell in three dimensions at the original frequency $f_{0}$ and (ii) the evolution of the trap when the excitation frequency is slightly shifted away from $f_{0}$.

\section{B. 3D cell trapping}

The 2D trapping capability of the SIDTs for cells has recently been verified both theoretically and experimentally [52]. Here we investigate the 3D trapping capabilities. The simulations are performed for a cell immersed in water and insonified by an acoustical vortex synthesized at the driving frequency $f_{0}=40 \mathrm{MHz}$ by a binary spiraling hologram. The maximum pressure amplitude in the focal plane $(z=0 \mathrm{~mm})$ is $3.4 \mathrm{MPa}$. The cell is assimilated to a spherical liquid scatterer whose acoustical properties are provided in Table I and correspond to typical values provided in Ref. [60].

The computed normalized lateral acoustic radiation force (ARF) versus $x$ and axial ARF versus $z$ for a cell with different radii $a=1,5,9,13$, and $17 \mu \mathrm{m}$ are calculated and represented in Figs. 2(a) and 2(b), respectively. Note
TABLE I. Acoustic properties.

\begin{tabular}{lccc}
\hline \hline Material & $\begin{array}{c}\text { Density } \rho \\
\left(\mathrm{kg} / \mathrm{m}^{3}\right)\end{array}$ & $\begin{array}{c}\text { Longitudinal speed of } \\
\text { sound } c_{l}(\mathrm{~m} / \mathrm{s})\end{array}$ & $\begin{array}{c}\text { Shear speed of } \\
\text { sound } c_{t}(\mathrm{~m} / \mathrm{s})\end{array}$ \\
\hline Cell & 1100 & 1508 & $\ldots$ \\
Pyrex & 2230 & 5640 & 3280 \\
Water & 1000 & 1500 & $\ldots$ \\
\hline \hline
\end{tabular}

that the lateral force is computed at the axial trap equilibrium position $z_{\text {trap }}$. In addition, the effective restoring forces versus cell radii $a$ in the lateral and axial directions are represented in Figs. 2(c) and 2(d). The effective restoring force is the smallest absolute value of the two positive and negative radiation force extrema located at two sides of the trapping position. These graphs show that the SIDT-based HATs produce a lateral trap for cells with radius up to $a=17 \mu \mathrm{m}(0.45 \lambda)$, even larger than the radius of the maximum first ring $(0.36 \lambda)$ in the lateral direction, while an axial trap is obtained for radii up to $a=13 \mu \mathrm{m}$ $(0.35 \lambda)$. That is, the designed HATs produce a $3 \mathrm{D}$ radiation trap of the considering typical cell with radius up to $\min \{0.35 \lambda, 0.45 \lambda\}=0.35 \lambda$. Note that in Fig. $2(b)$, the axial trapping position is shifted away from the source plane when the size of the cell is increased. Indeed, for small cells (see $a=1,5$, and $9 \mu \mathrm{m}$ ), the gradient force is dominant and the scattering force is negligible [61]. So the cells are trapped at or near the focal point. When the radius of the cell is increased, the scattering force can no longer be neglected and will push the cell along the propagation direction. The restoring force nevertheless remains sufficient to balance the pushing force for cells smaller than (a)

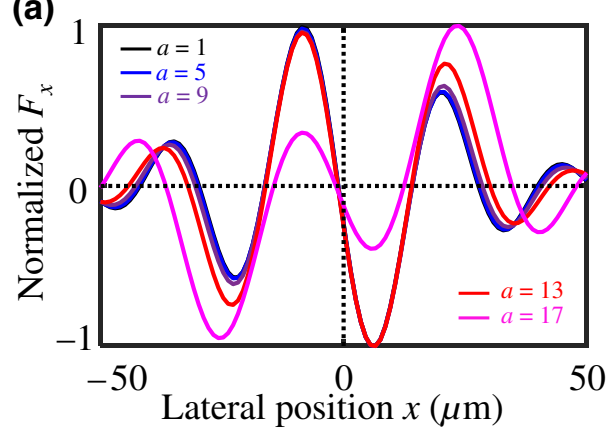

(c)

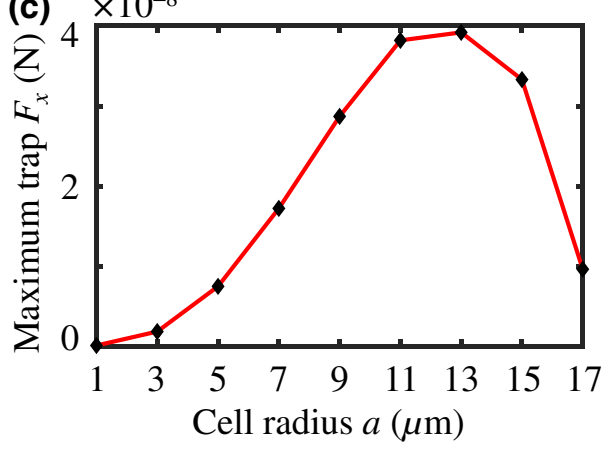

(b)

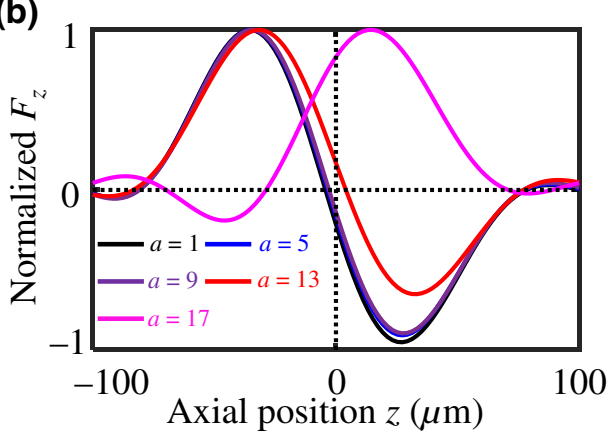

(d)

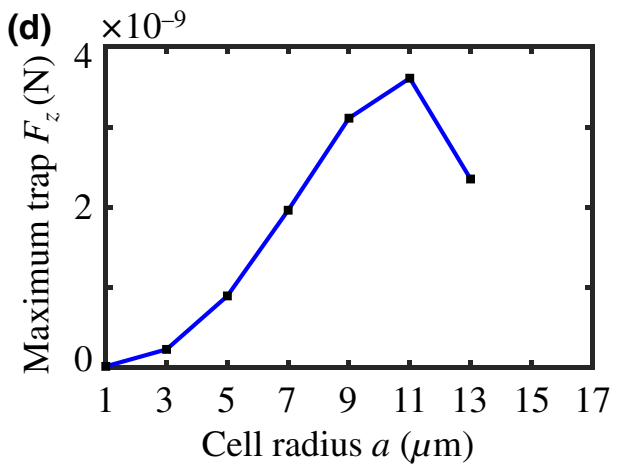

FIG. 2. Three-dimensional cell trapping with a spiraling one-sided HAT driven at the frequency $f_{0}=$ $40 \mathrm{MHz}$. The cell is modeled as a liquid sphere with typical acoustical parameters given in Table I. (a) The normalized lateral force versus $x$ position (at axial equilibrium position) and (b) the normalized axial force versus $z$ position for cells with different radii $a$. The radius of the first ring of maximum intensity of the acoustical vortex is $13.6 \mu \mathrm{m}$ [see Fig. 1(c)], corresponding to $0.36 \lambda$. (c) The maximum lateral and (d) axial trapping (effective restoring) forces for cells with radii ranging from $a=1$ to $17 \mu \mathrm{m}$. Only the stable trapping sizes are represented. The axial trap is lost for cell radii $a=15$ and $17 \mu \mathrm{m}$. 

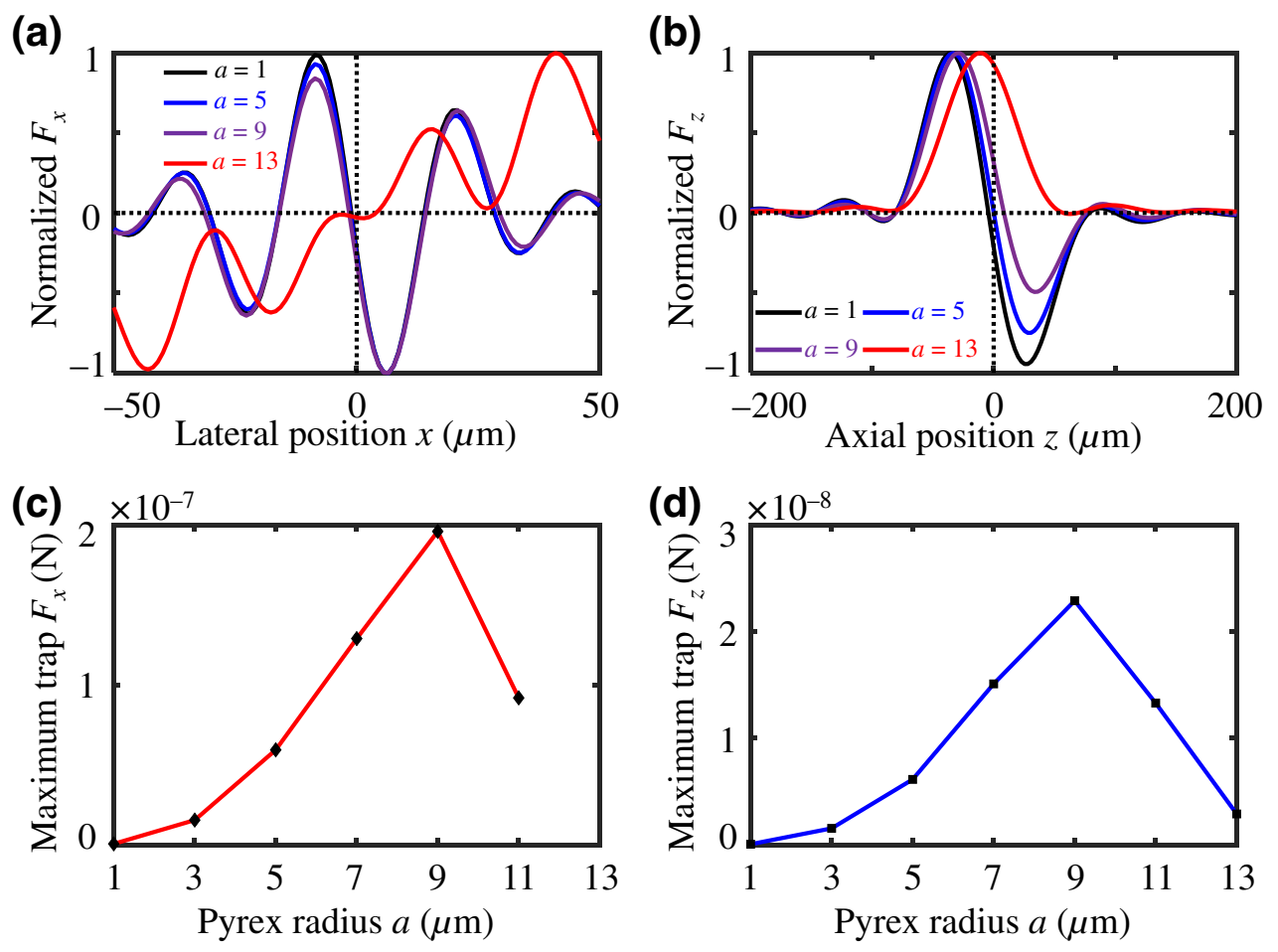

FIG. 3. Three-dimensional Pyrex microparticle trapping with a vortex-based one-sided HAT at the designed frequency $f_{0}=40 \mathrm{MHz}$ with different radii $a$. The Pyrex is modeled as an elastic sphere without consideration of the viscosity. The acoustical parameters are given in Table I. (a) The normalized lateral force versus $x$ (at the axial equilibrium position) and (b) the normalized axial force versus $z$ position for particle radii $a=1,5,9$, and $13 \mu \mathrm{m}$. (c),(d) The maximum trapping (effective restoring) force for particle radii ranging from $a=1$ to $13 \mu \mathrm{m}$. The lateral trapping is lost for Pyrex radius $a=13 \mu \mathrm{m}$ at the axial equilibrium position $z_{\text {trap }}=57.5 \mu \mathrm{m}$.
$0.35 \lambda$, but the equilibrium position is shifted towards the beam direction of propagation (see $a=13$ and $17 \mu \mathrm{m}$ ). When the radius is further increased, the cell will escape from the trap.

Note that the axial trapping forces calculated here for a realistic acoustic field are several orders of magnitude larger than the force resulting from the balance between gravity and buoyancy $\left[4.2 \times 10^{-15}\right.$ Newton $(\mathrm{N})$ for radius $a=1 \mu \mathrm{m}$, and $9.2 \times 10^{-12} \mathrm{~N}$ for radius $\left.a=13 \mu \mathrm{m}\right]$. In a flowing microchannel configuration, additional drag forces could also affect the trapping. Hence, it is insightful to compare the acoustic radiation force to the drag force magnitude. For typical velocities in a microchannel of $v_{f}=5$ to $500 \mu \mathrm{m} / \mathrm{s}[62,63]$, the drag force $F_{d}=$ $6 \pi \mu a v_{f}$ (with $\mu$ the dynamic viscosity) applied to a cell of radius $a=11 \mu \mathrm{m}$ lies in the order of 1 to $100 \mathrm{pN}$, which remains significantly smaller than the acoustic radiation force computed here for a pressure level of 3.4 $\mathrm{MPa}$ in the focal plane. Note that in Ref. [52] cells were moved (at lower pressure levels) at maximum velocities of $1.2 \mathrm{~mm} \mathrm{~s}^{-1}$, which corresponds to trapping forces of the order of $200 \mathrm{pN}$. These calculations and experiments show that the acoustic radiation force is sufficient to manipulate cells in flowing microchannels at moderate flow rates.

\section{Three-dimensional Pyrex trapping}

In this section, we investigate $3 \mathrm{D}$ trapping of spherical Pyrex particles with radii from $a=1$ to $a=13 \mu \mathrm{m}$ in water with the same acoustic field as that used in the previous section for cell trapping. Since Pyrex support both longitudinal and transverse waves, an elastic scattering model is considered [46,61]. The acoustic properties of Pyrex used for the simulations are given in Table I. The normalized lateral and axial radiation forces versus the respective positions are given in Figs. 3(a) and 3(b), with the maximum lateral and axial trapping (restoring) forces versus particle radii $a$ given in (c) and (d), respectively. These results show 3D Pyrex particle trapping for radii up to $a=11 \mu \mathrm{m}[0.29 \lambda$; see Figs. 3(c) and 3(d)], with a maximum for both lateral and axial trapping obtained around $a=9 \mu \mathrm{m}$. It is interesting to note that the lateral trapping at the axial equilibrium position $z_{\text {trap }}$ is lost for $a=$ $13 \mu \mathrm{m}$ since the axial trapping position is far away $\left(z_{\text {trap }}=\right.$ $57.5 \mu \mathrm{m})$ from the designed focus by the large pushing scattering force. Similarly to cells, the gravity effect on a Pyrex sphere is negligible compared to the radiation force since the balance between gravity and buoyancy leads to net forces of $5.2 \times 10^{-14} \mathrm{~N}$ for a radius of $a=1 \mu \mathrm{m}$, and $6.9 \times 10^{-11} \mathrm{~N}$ for a radius of $a=11 \mu \mathrm{m}$.

Note that the influence of the aperture on the 3D trapping capabilities of the tweezers for both Pyrex particles and cells are discussed in Appendix C.

\section{AXIAL DISPLACEMENT OF THE TRAP BY FREQUENCY TUNING}

\section{A. Axial displacement of the focal point}

The 3D trapping ability of spherical focused vortices produced by HATs has been verified in Sec. III. Now we investigate what happens to the trapping equilibrium position when the actuation frequency of the HAT is tuned, 


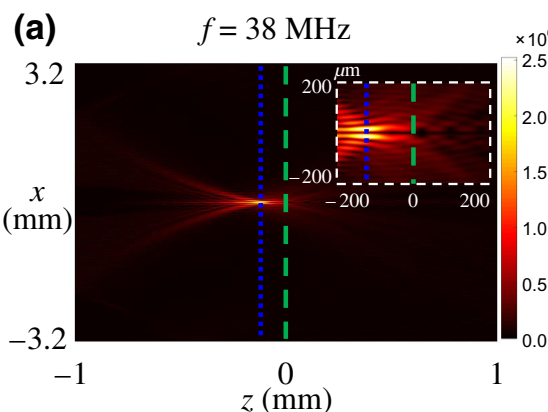

(b)

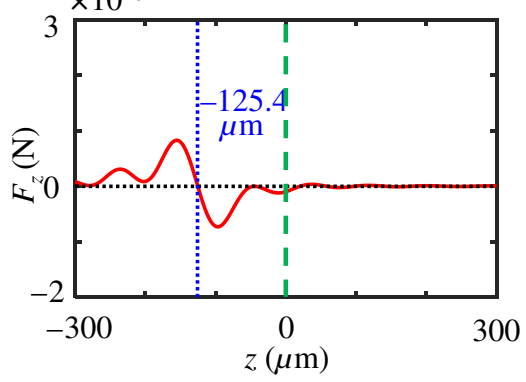

(c)

$f=40 \mathrm{MHz}$

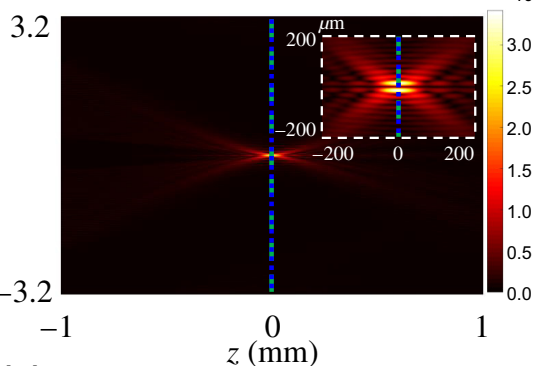

(d)

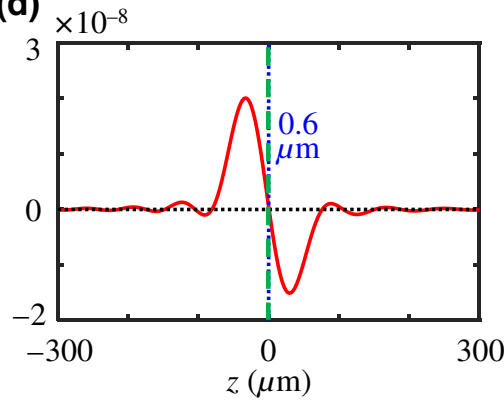

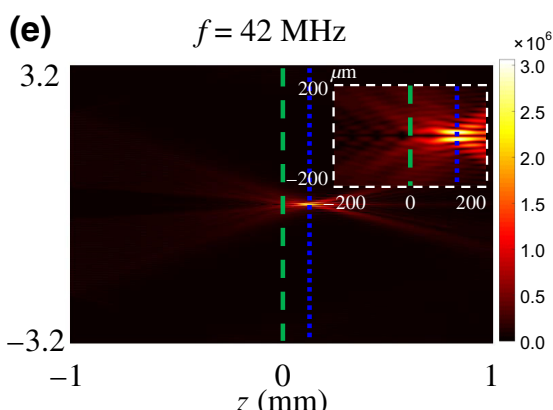

(f)

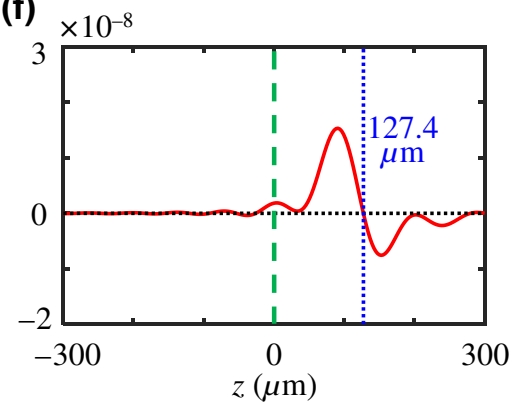

FIG. 4. (a),(c),(e) The acoustic pressure amplitude in the propagation plane $(x, y=0, z)$ with different activated frequencies $f=38$, 40, and $42 \mathrm{MHz}$, respectively. Enlarged insets including the focal plane are shown in the top right corners. (b),(d),(f) The axial radiation force versus different $z$ positions for a Pyrex particle with radius $a=7 \mu \mathrm{m}$. The Pyrex particle can be trapped in three dimensions (the lateral force versus $x$ position is not shown here for brevity) and the axial equilibrium position is changed with different activated frequencies. The green dashed line describes the originally designed axial focal position $(z=0)$ at $f_{0}=40 \mathrm{MHz}$, while the blue dotted line indicates the actual axial position of the focal plane. The effective restoring force decreases when the excitation frequency deviates away from $f_{0}$, e.g., $f=38$ and $42 \mathrm{MHz}$.

while keeping the geometric shapes of the IDTs identical. Indeed, recent airborne investigations by Muelas-Hurtado et al. [53] revealed that slight frequency tuning of the excitation of a spiraling HAT induce an axial translation of the position of the focal plane, combined with a distortion of the beam since the equations of the Archimedes-Fermat spirals [51] are not invariant by a change in the tuned frequency. However, they did not study the trapping capabilities of these beams and whether the distortion remains sufficiently limited to maintain a 3D trap.

As illuminated in Fig. 1(a), the focal plane is moved toward the source plane (i.e., focal depth decreases) when the excitation frequency $f<f_{0}$ [see Fig. 4(a) for $f=$ $38 \mathrm{MHz}$ ], and away from it when $f>f_{0}$ [see Fig. 4(e) for $f=42 \mathrm{MHz}]$.

\section{B. Axial displacement of Pyrex particles}

In this section, radiation force simulations are conducted for a Pyrex spherical particle of radius $a=7 \mu \mathrm{m}$, so that the lateral and axial trapping forces are large and the restoring force around the equilibrium position is more symmetrical (compared to the case $a=9 \mu \mathrm{m}$ ). The axial radiation force versus axial position $z$ near the designed focus $(z=0)$ for the excitation frequencies $f=38,40$, and $42 \mathrm{MHz}$ are represented in Figs. 4(b), 4(d), and 4(f), respectively. The Pyrex sphere can be trapped axially at the new focal plane at $z_{\text {trap }}=-125.4 \mu \mathrm{m}$ for $f=38 \mathrm{MHz}$, and at $z_{\text {trap }}=127.4 \mu \mathrm{m}$ for $f=42 \mathrm{MHz}$. The axial translation of the trapping point for these two cases is of the order of $18 a$, which is large enough for axial control of a particle in a microchannel. Lateral trapping at the axial equilibrium position $z_{\text {trap }}$ (not shown here for the sake of brevity) is also fulfilled since the main energy is localized near the focal plane with a large intensity gradient. Hence, these simulations demonstrate controllable axial displacement of trapped Pyrex particles when tuning the excitation frequency.

We further conducted a systematic analysis of the axial translation of the trapping positions and effective restoring forces versus the excitation frequency within the regime $f=[32,45] \mathrm{MHz}$ [see Figs. 5(a) and 5(b), respectively]. When the excitation frequency is close to the designed frequency $f_{0}=40 \mathrm{MHz}$, the trapping position $z_{\text {trap }}$ linearly depends on the excitation frequencies (see the regime of $[37,43] \mathrm{MHz}$ ), while outside this range, the trend is no longer linear due to a large distortion of the acoustic vortex. Meanwhile, the effective restoring force $F_{z}$ is maximum at the designed frequency $f_{0}=40 \mathrm{MHz}$, and then drops rapidly and nearly symmetrically when the excitation frequency is larger or smaller than $f_{0}$, down to less than $1 / 6$ of its maximum value outside the $[37,43] \mathrm{MHz}$ range. Hence, the range of interest for practical application (linear and hence predictable displacement and sufficient 
(a)

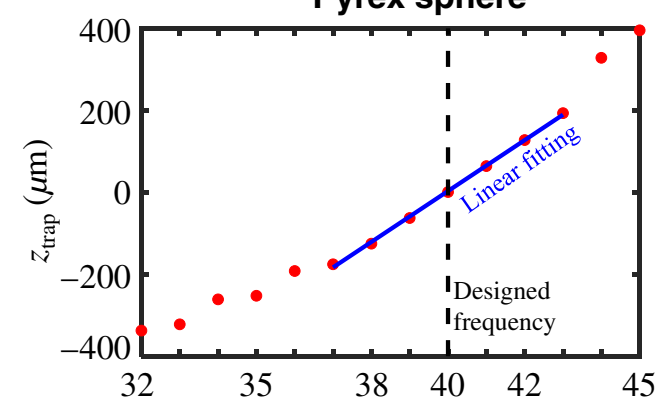

(c)

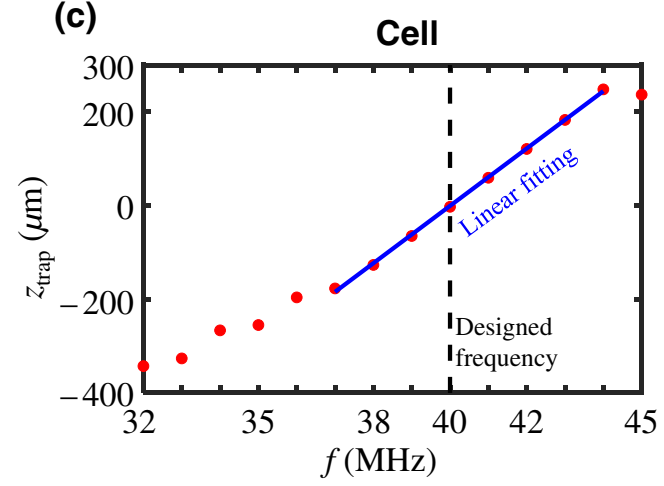

(b)

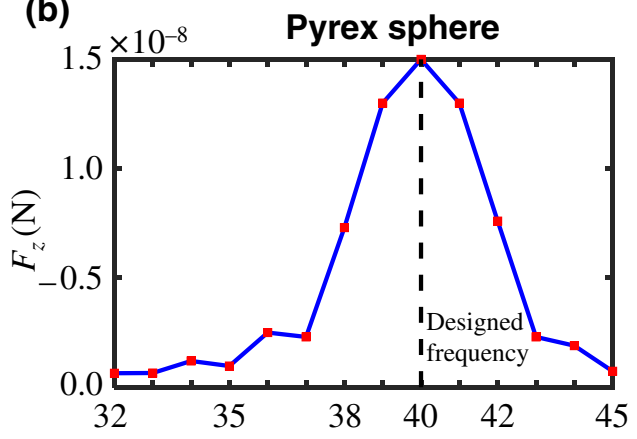

(d)

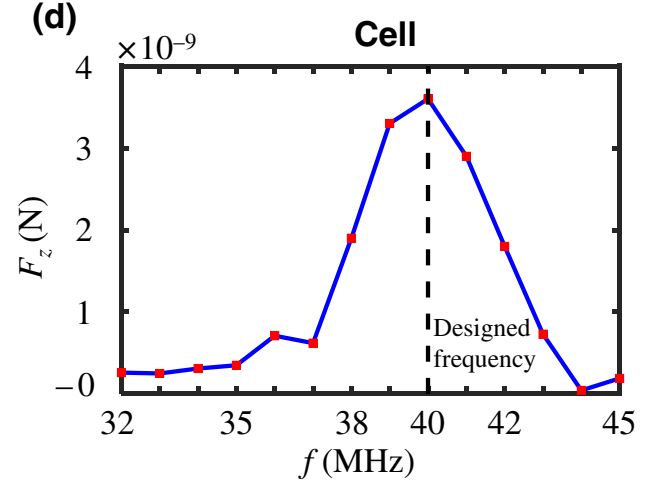

FIG. 5. The axial trapping positions $\left(z_{\text {trap }}\right)$ along the $z$ direction and the effective restoring force $F_{z}$ versus excitation frequencies for (a),(b) Pyrex spherical particles with radius $a=7 \mu \mathrm{m}$ and (c),(d) cells with $a=$ $11 \mu \mathrm{m}$. The excitation frequencies are selected near the designed frequency $f_{0}=$ $40 \mathrm{MHz}$ from $f=32 \mathrm{MHz}$ to $f=45 \mathrm{MHz}$. In the regime of $[37,43] \mathrm{MHz}$ for (a) the Pyrex sphere, and of $[37,44] \mathrm{MHz}$ for (c) the cell, the axial trapping position $z_{\text {trap }}$ agrees exactly with the linear fitting (blue solid line). The effective restoring forces $F_{z}$ drop when the excitation frequencies are away from $f_{0}$. axial trapping force) is the $[37,43] \mathrm{MHz}$ interval. In this range, the displacement is about $400 \mu \mathrm{m}$, that is, more than 100 times the size of the particle, and 10 times the wavelength.

\section{Axial displacement of cells}

We conducted a similar analysis for cells with radius $a=11 \mu \mathrm{m}$ [see Figs. 5(c) and 5(d)]. The analysis shows that cells can also be moved, while keeping a 3D trap when the frequency is tuned in the range $f=[32,45] \mathrm{MHz}$. This leads to a maximum displacement range of about $650 \mu \mathrm{m}$ with a linear trend observed in the regime $f=[37,44]$ $\mathrm{MHz}$, which is more than 57 times the size of the cell, and 16 times the wavelength. The trapping radiation force is maximum at the designed frequency $f_{0}=40 \mathrm{MHz}$ and drops with the excitation frequency away from $f_{0}$, which is similar to the Pyrex particle case.

\section{CONCLUSION AND PERSPECTIVES}

This work theoretically demonstrates the ability to obtain a 3D acoustic radiation trap for both elastic microparticles and cells with realistic acoustic fields mimicking that produced with spiraling interdigitated transducers. This extends to three dimensions the manipulation possibilities offered by spiraling interdigitated transducers $[23,51,52]$. In addition, we also demonstrate that the positions of the 3D trapped cells and microparticles can be moved axially in a predictable manner by tuning the excitation frequency near the original frequency used for the design of the spiraling transducers. The magnitude of the displacement that can be achieved is 2 orders of magnitude larger than the particle radius and more than an order of magnitude larger than the wavelength. This opens possibilities for axial displacement of the trapped particle by a simple shift of the driving frequency without any moving part. This is critical since, for manipulation in microchannels, the tweezers cannot be moved axially. The next steps include (i) experimental verification of these predictions and (ii) investigation of the role played by Eckart streaming [64] and the resulting drag, especially for particles with low acoustic contrast such as cells. From an experimental perspective, some holographic acoustical tweezers based on the Archimedes-Fermat spiral have already been studied in Ref. [52] at $40 \mathrm{MHz}$. The main challenge to obtaining unequivocal results on 3D trapping and axial manipulation is performing the experiments in a large chamber with absorbing walls to avoid spurious reflections by the walls that could affect the acoustic trap.

Another interesting path would be to investigate fields with optimized properties for 3D trapping and frequency shift driven displacement. Indeed, acoustical vortices combine many interesting properties: they can be synthesized with a $2 \mathrm{D}$ phase hologram, enable $3 \mathrm{D}$ trapping, and, as shown in this paper, enable $z$ displacement by switching the active hologram driving frequency. But they also have some drawbacks, such as an axial trapping force one order of magnitude weaker than the the lateral force. Some other types of beam have been studied in the literature for 3D trapping, such as bottle beams (see, e.g., Refs. [47,65] in acoustics). These beams exhibit, on the opposite, larger axial force and weaker lateral force. Marzo et al. [47] also 
conducted some optimization procedure to determine optimal fields in the long wavelength regime. But the design of the optimal acoustic field for 3D selective particle trapping beyond this regime still remains an open problem. Also, the addition of interesting frequency-driven displacement properties to a static hologram essentially remains unexplored.

\section{ACKNOWLEDGMENTS}

We acknowledge support from the ERC Generator, Prematuration programs, and Talent project funded by ISITE-ULNE and from Institut Universitaire de France.

\section{APPENDIX A: ANGULAR SPECTRUM METHOD}

The schematic to understand how to use ASM to calculate the wave propagation in free space is given in Fig. 6. Assume that we know the acoustic pressure field in the source plane or a plane parallel to the source plane, then angular spectrum $\left.S\left(k_{x}, k_{y}\right)\right|_{z=0}$ (e.g., at source plane) is easily obtained by using the $2 \mathrm{D}$ fast Fourier transform inverse fast Fourier transform (IFFT), which is related to the ASM-based beam shape coefficients $H_{n m}$ [see Eq. (2)]. To calculate the radiation force according to Eqs. (4a)-(4c), it is convenient to expand the incident beam at the origin of the scatter $\left(x_{s}, y_{s}, z_{s}\right)$. The propagation of the acoustic field from the source to the scatter plane is calculated by using

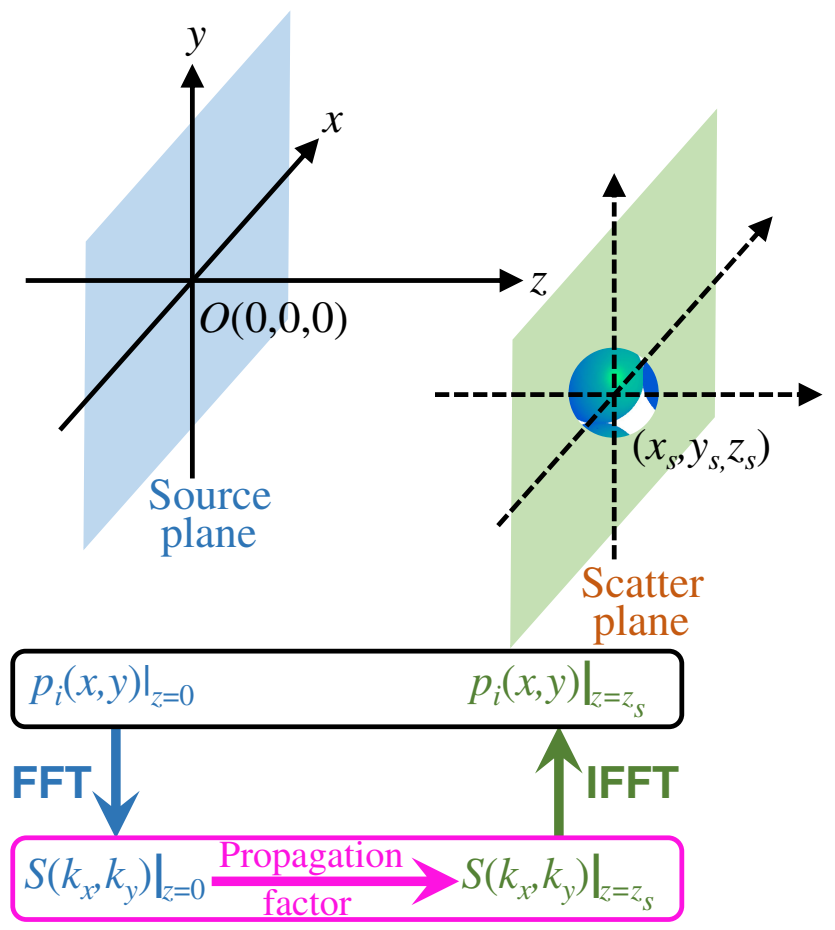

FIG. 6. Flowchart of the angular spectrum method. The acoustic pressure in the black box lies in real physical space and can be measured in experiment, while the angular spectrum in the magenta box lies in Fourier space. the propagation factor in Fourier space:

$$
\left.S\left(k_{x}, k_{y}\right)\right|_{z=z_{s}}=\left.S\left(k_{x}, k_{y}\right)\right|_{z=0} e^{i k_{x} x_{S}+i k_{y} y_{s}+i \sqrt{k^{2}-k_{x}^{2}-k_{y}^{2}} z_{S}} .
$$

This can be used to calculate the ASM-based beam shape coefficients $H_{n m}$ at the scatterer center and further the 3D radiation force. Note that in this paper the viscous attenuation of the wave is not considered in the angular spectrum propagator. At the working frequency $(40 \mathrm{MHz})$ in water at room temperature, the acoustic attenuation length [66] $L_{a}=2 \rho_{0} c_{0}^{3} /\left[\omega^{2}\left(4 / 3 \mu_{s}+\mu_{b}\right)\right] \sim 26 \mathrm{~mm}$, which is much larger than the designed focal depth (about $1 \mathrm{~mm}$ ). Since the wave amplitude decreases with the propagation distance as $e^{-z / L_{a}}$, the pressure magnitude will lose about $3.8 \%$ amplitude between the source and the focal plane, which is significant but does not modify the orders of magnitude presented in this paper. Note also that the real pressure field, including the amplitude and phase at the scatter plane, can be obtained by using the 2D inverse Fourier transform (IFFT) of the angular spectrum $\left.S\left(k_{x}, k_{y}\right)\right|_{z=z_{s}}$.

\section{APPENDIX B: PRINCIPLE OF FOCUSED-VORTEX-BASED ACOUSTICAL TWEEZERS}

It is difficult to produce an ideal spherical vortex in experiment since the transducers need to be placed all over the $4 \pi$ steradians enclosing the manipulation area [61]. However, this can be overcome by using a focused spherical vortex with finite aperture (steradian less than $2 \pi)[45,51,52,67]$. Similarly to the plane standing waves consisting of two counterpropagating traveling waves, a spherical Bessel beam can be produced by the interference of converging and diverging spherical Hankel beams. This agrees with the mathematical fact that a Bessel function can be written as the addition of Hankel functions of the first and second kinds (with a ratio of 1/2). To coincide with the radiation force derivations $[44,56]$, the time harmonic here is chosen as $e^{(-i \omega t)}$ so that the converging Hankel vortex is described by the spherical Hankel function of the second kind $h_{n}^{(2)}$. An ideal converging Hankel spherical vortex is described in spherical coordinates $(r, \theta, \varphi)$ as [51]

$$
p^{*}(r, \theta, \varphi)=p_{0} h_{n}^{(2)}(k r) P_{n}^{m}(\cos \theta) e^{i(m \varphi-\omega t)},
$$

where $p_{0}$ is the wave amplitude and the $P_{n}^{m}$ are the associated Legendre functions of order $(n, m)$. Following the same procedure as in Ref. [51] and using the farfield asymptotic expression $h_{n}^{(2)}(k r) \simeq i^{(n+1)} e^{-i k r} /(k r)$, the geometrical shapes of the two spiral electrodes can be 
described in cylindrical coordinates $(R, \varphi, z)$ as

$$
\begin{aligned}
& R_{1}=\frac{1}{k} \sqrt{\left(\varphi+C_{2}\right)^{2}-(k z)^{2}} \\
& R_{2}=\frac{1}{k} \sqrt{\left(\varphi+C_{2}+\pi\right)^{2}-(k z)^{2}}
\end{aligned}
$$

where $m=1$ is for the first-order vortex and $C_{2}$ is a constant. The geometrical shapes described by $R_{1}$ and $R_{2}$ [see, e.g., Fig. 7(a)] are obtained by extracting the intersection between the Hankel beam isophase surfaces and a plane. The phase shift $\pi$ is applied here for the two isophase shapes $R_{1}$ and $R_{2}$ since it is easy to achieve by a piezoelectric transducer with positive and negative electrodes. If we set a focal depth $(z)$ for a given frequency (or wave number $k$ ), the aperture radius and angle can be defined by choosing appropriate azimuthal angle regimes $\varphi$ (also the number of spiral turn $N$ ) according to Eqs. (B2) and (B3).

\section{APPENDIX C: ROLE PLAYED BY THE APERTURE}

In the expressions of the two spiral electrodes given in Eqs. (B2) and (B3), the minimum and maximum azimuthal angles $\varphi_{\min }$ and $\varphi_{\max }$, which determine the aperture angle of the HAT, can be chosen arbitrarily. For manipulation in microscopes, the minimum angle can be chosen to leave some space for observation near the central axis since generally the electrodes are not transparent [51]. The maximum azimuthal angle proportional to the number of spiral turns plays an important role in the axial and lateral trapping capabilities. Here, lateral and axial trapping forces for different numbers of spiral turns with the corresponding aperture radii and angles are given in Table II and three selected patterns are given in Fig. 7(a). The axial radiation force versus the axial position $z$ for a cell with radius $a=11 \mu \mathrm{m}$ is given in Fig. 7(b), and for a Pyrex sphere with $a=9 \mu \mathrm{m}$ in Fig. 7(c). The axial trapping force increases with increasing aperture angle. For all the numbers of turns discussed, the cell can be trapped in three

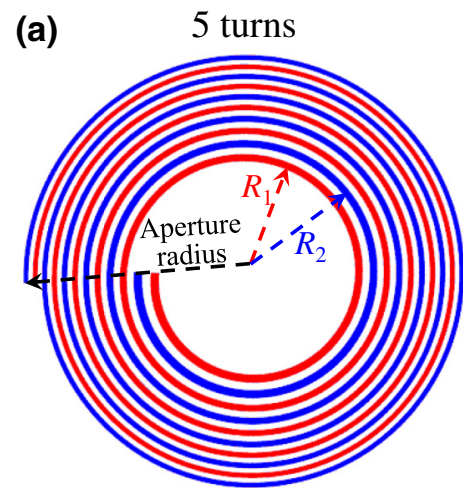

(b)

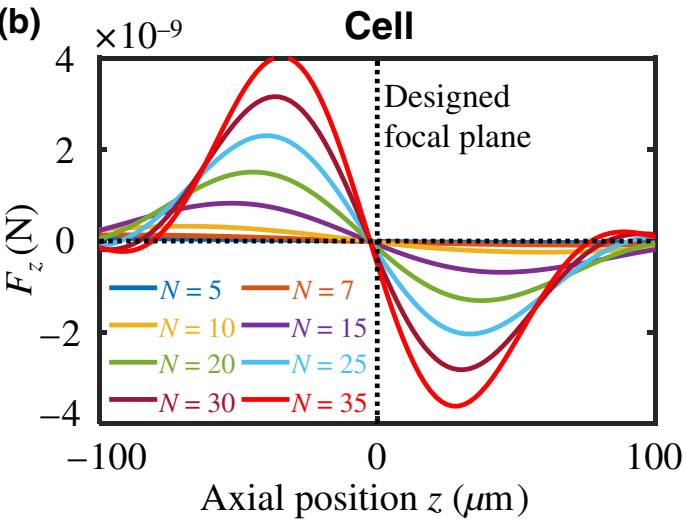

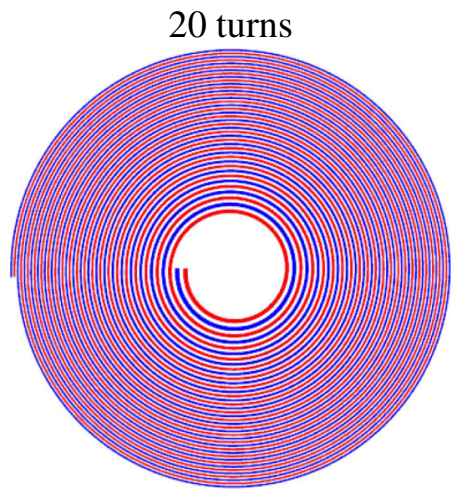

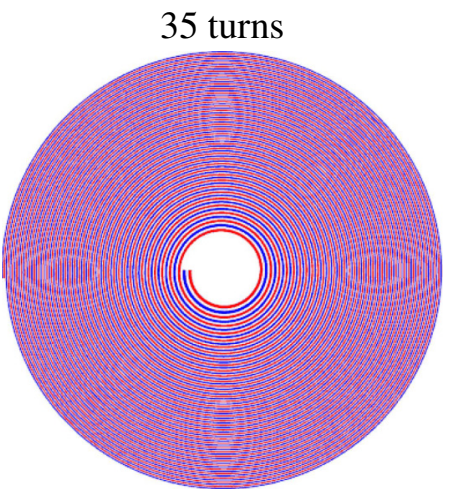

(c)

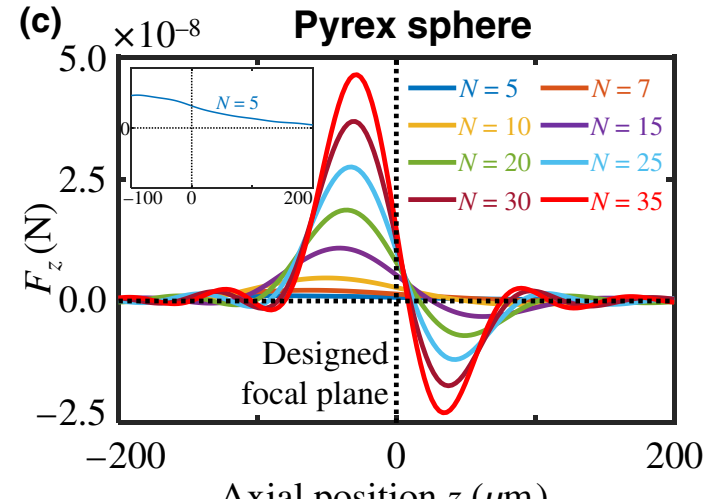

FIG. 7. (a) Geometric shapes of spiral electrodes (IDTs) with different numbers of turns. The aperture radius is the maximum radius of $R_{1}, R_{2}$, as indicated by the black dashed line. The aperture angle is computed by the arctangent value of the aperture radius over the designed focal depth. The axial radiation force $F_{z}$ versus different positions $z$ for (b) a cell with radius $a=11 \mu \mathrm{m}$ and (c) a Pyrex sphere with $a=9 \mu \mathrm{m}$, with a focused-vortex-based acoustical tweezer at frequency $f_{0}=40 \mathrm{MHz}$ with different numbers of spiral turns. The scattering force contribution is relatively strong for a Pyrex sphere (elastic model) than for a cell (liquid model). The axial trapping positions for the cell are almost the same, while those for the Pyrex move closer to the designed focal position as the number of spiral turns increase, leading to the increase of focused ability (hence, gradient force). The gravity effect could be ignored compared to the trapping force. 
TABLE II. Number of spiral turns $N$ for acoustical tweezers with the same focal length $1 \mathrm{~mm}$ and related aperture angles.

\begin{tabular}{lcccccccc}
\hline \hline & \multicolumn{8}{c}{$N$} \\
\cline { 2 - 8 } & 5 & 7 & 10 & 15 & 20 & 25 & 30 & 35 \\
\hline $\begin{array}{c}\text { Aperture radius } \\
(\mathrm{mm})\end{array}$ & 0.74 & 0.86 & 1.02 & 1.27 & 1.50 & 1.72 & 1.94 & 2.15 \\
$\begin{array}{c}\text { Aperture angle } \\
(\mathrm{deg})\end{array}$ & 36 & 41 & 46 & 52 & 56 & 60 & 63 & 65 \\
\hline \hline
\end{tabular}

dimensions, while for the Pyrex sphere, the axial trap is lost for the case with five turns [see the enlarged inset in Fig. 7(c)]. Indeed, the acoustic impedance of cells is smaller than that of Pyrex, leading to a weaker scattering contribution that will push the cell or particle outside of the trap center. In addition, the axial trapping position $z_{\text {trap }}$ moves closer to the designed focal plane when the number of turns increases. This is because the restoring gradient force is enhanced more than the pushing scattering force.

[1] L. Rayleigh, On the pressure of vibration, Philos. Mag. 3, 338 (1902).

[2] L. Rayleigh, On the momentum and pressure of gaseous vibrations, and on the connection with the virial theorem, Philos. Mag. 10(57), 366 (1905).

[3] P. Biquard, Les ondes ultra-sonores, Rev. D’Acous. 1, 93 (1932).

[4] P. Biquard, Les ondes ultra-sonores II, Rev. D’Acous. 1, 315 (1932).

[5] L. Brillouin, Sur les tensions de radiation, Ann. Phys. 4, 528 (1925).

[6] L. Brillouin, Les tensions de radiation; leur interprétation en mécanique classique et en relativité, J. Phys. Radium 6, 337 (1925).

[7] L. V. King, On the acoustic radiation pressure on spheres, Proc. R. Soc. London 147, 212 (1934).

[8] K. Yosika and Y. Kawasima, Acoustic radiation pressure on a compressible sphere, Acustica 5, 167 (1955).

[9] L. Gor'ov, On the forces acting on a small particle in an acoustic field in an ideal fluid, Sov. Phys. Dokl. 6, 773 (1962).

[10] R. Boyle, Ultrasonics, Sci. Prog. 23, 75 (1928).

[11] R. Boyle, G. Taylor, and D. Froman, Cavitation in the track of an ultrasonic beam, R. Soc. Can. Trans. 23, 187 (1929).

[12] C. Allen and I. Rudnick, A powerfull high frequency siren, J. Acoust. Soc. Am. 19, 857 (1936).

[13] S. Tran, P. Marmottant, and P. Thibault, Fast acoustical tweezers for the two-dimensional manipulation of individual particles in mocrofluidic channels, Appl. Phys. Lett. 101, 114103 (2012).

[14] X. Ding, S.-C. Lin, B. Kiraly, H. Yue, S. Li, I.-K. Chiang, J. Shi, S. J. Benkovic, and T. J. Huang, On-chip manipulation of single microparticles, cells and organisms using surface acoustic waves, Proc. Natl. Acad. Sci. 109, 11105 (2012).

[15] K. Melde, A. Mark, T. Qiu, and P. Fischer, Holograms for acoustis, Nature 537, 518 (2016).
[16] Y. Ochiai, T. Hoshi, and J. Rekimoto, Three dimensional mid-air acoustic manipulation by ultrasonic phased arrays, PLoS ONE 9, e97590 (2014).

[17] Y. Ochiai, T. Hoshi, and J. Rekimoto, Pixie dust: Graphics generated by levitated and animated objects in computational acoustic-potential field, ACM Trans. Graphics 33, 1 (2014).

[18] A. Marzo and B. Drinkwater, Holographic acoustic tweezers, Proc. Natl. Acad. Sci. USA 116, 84 (2019).

[19] R. Hirayama, D. Plasencia, N. Masuda, and S. Subramanian, A volumetric display for visual, tactile and audio presentation using acoustic trapping, Nature 575, 320 (2019).

[20] Z. Ma, A. Melde, K. Athanassiadis, M. Shau, H. Richter, T. Qiu, and P. Fischer, Spatial ultrasound modulation by digitally controlling microbubble arrays, Nat. Commun. 11, 4537 (2020).

[21] J. Wu, Acoustical tweezers, J. Acoust. Soc. Am. 89, 2140 (1991).

[22] A. Ashkhin, J. Dziedzic, J. Bjorkholm, and S. Chu, Observation of a single-beam gradient force optical trap for dielectric particles, Opt. Lett. 11, 288 (1986).

[23] M. Baudoin and J.-L. Thomas, Acoustical tweezers for particle and fluid micromanipulation, Annu. Rev. Fluid Mech. 52, 205 (2020).

[24] J. Lee, S.-Y. Teh, A. Lee, H. Kim, C. Lee, and K. Shung, Single beam acoustic trapping, Appl. Phys. Lett. 95, 073701 (2009).

[25] Z. Gong and M. Baudoin, Single beam acoustical tweezers based on focused beams: A numerical analysis of 2D and 3D trapping capabilities (submitted).

[26] B. Hefner and P. Marston, An acoustical helicoidal wave transducer with applications for the alignment of ultrasonic and underwater systems, J. Acoust. Soc. Am. 106, 3313 (1999).

[27] D. Baresch and T. J.-L. R. Marchiano, Three-dimensional acoustic radiation force on an arbitrary located elastic sphere, J. Acoust. Soc. Am. 133, 25 (2013).

[28] C. Courtney, B. Drinkwater, C. Demore, S. Cochran, and A. Grinenko, Dexterous manipulatino of microparticles using bessel-function acoustic pressure fields, Appl. Phys. Lett. 12, 123508 (2013).

[29] J. Thomas and R. Marchiano, Pseudo Angular Momentum and Topological Charge Conservation for Nonlinear Acoustical Vortices, Phys. Rev. Lett. 91, 244302 (2003).

[30] Y. Yang, T. Ma, S. Li, Q. Zhang, J. Huang, Y. Liu, J. Zhuang, Y. Li, X. Du, L. Niu, et al., Self-navigated 3d acoustic tweezers in complex media based on time reversal, Research 2021, 9781394 (2021).

[31] A. Riaud, J. Thomas, E. Charron, A. Bussonière, and O. Bou Matar, Anisotropic Swirling Surface Acoustic Waves from Inverse Filtering for On-Chip Generation Of Acoustic Vortices, Phys. Rev. Appl. 4, 034004 (2015).

[32] A. Riaud, J. Thomas, M. Baudoin, and O. Bou Matar, Taming the degeneration of bessel beams at anisotropic-istropic interface: Toward three-dimensional control of confined vortical waves, Phys. Rev. E 92, 062301 (2015).

[33] A. Riaud, M. Baudoin, J. Thomas, and O. Bou Matar, Saw synthesis with IDTs array and the inverse filter: Toward a versatile saw toolbox for microfluidics and biological applications, IEEE Trans. Ultrason. Ferroelectr. Freq. Control 63, 1601 (2016). 
[34] N. Jiménez, R. Picó, V. Sánchez-Morcillo, V. RomeroGarcía, L. García-Raffi, and K. Staliunas, Formation of high-order acoustic bessel beams by spiral diffraction gratings, Phys. Rev. E 12, 014016 (2019).

[35] T. Wang, M. Ke, W. Li, Q. Yang, C. Qiu, and Z. Liu, Particle manipulation with acoustic vortex beam induced by a brass plate with spiral shape structure, Appl. Phys. Lett. 109, 123506 (2016).

[36] X. Jiang, J. Zhao, S. Liu, B. Liang, X. Zou, J. Yang, C. Qiu, and J. Cheng, Broadband and stable acoustic vortex emitter with multi-arm coiling slits, Appl. Phys. Lett. 108, 203501 (2016).

[37] M. Terzi, S. Tsysar, P. Yuldashev, M. Karzova, and O. Sapozhnikov, Generation of a vortex ultrasonic beam with a phase plate with an angular dependence of the thickness, Mosc. Univ. Phys. Bull. 72, 61 (2017).

[38] X. Jiang, Y. Li, B. Liang, and L. Zhang, Convert Acoustic Resonances to Orbital Angular Momentum, Phys. Rev. Lett. 117, 034301 (2016).

[39] A. Riaud, M. Baudoin, O. Bou Matar, L. Becera, and J. Thomas, Selective Manipulation of Microscopic Particles with Precursor Swirling Rayleigh Waves, Phys. Rev. Appl. 7, 024007 (2017).

[40] L. Le Brizoual, F. Sarry, O. Elmazria, P. Alnot, S. Ballandras, and T. Pastureaud, $\mathrm{GHz}$ frequency $\mathrm{ZnO} / \mathrm{Si}$ saw device, IEEE Trans. Ultrason. Ferroelectr. Freq. Control 55, 442 (2008).

[41] P. L. Marston, Axial radiation force of a bessel beam on a sphere and direction reversal of the force, J. Acoust. Soc. Am. 120, 3518 (2006).

[42] L. Zhang and P. L. Marston, Geometrical interpretation of negative radiation forces of acoustical bessel beams on spheres, Phys. Rev. E 84, 035601 (2011).

[43] X.-D. Fan and L. Zhang, Trapping Force of Acoustical Bessel Beams on a Sphere and Stable Tractor Beams, Phys. Rev. Appl. 11, 014055 (2019).

[44] Z. Gong, P. L. Marston, and W. Li, T-matrix evaluation of three-dimensional acoustic radiation forces on nonspherical objects in bessel beams with arbitrary order and location, Phys. Rev. E 99, 063004 (2019).

[45] D. Baresh, J.-L. Thomas, and R. Marchiano, Spherical vortex beams of high radial degree for enhanced single-beam tweezers, J. Appl. Phys. 113, 184901 (2013).

[46] D. Baresch, J.-L. Thomas, and R. Marchiano, Observation of a Single-Beam Gradient Force Acoustical Trap for Elastic Particles: Acoustical Tweezers, Phys. Rev. Lett. 116, 024301 (2016).

[47] A. Marzo, S. A. Seah, B. W. Drinkwater, D. R. Sahoo, B. Long, and S. Subramanian, Holographic acoustic elements for manipulation of levitated objects, Nat. Commun. 6, 8661 (2015).

[48] M. Ghanem, A. Maxwell, Y. Wang, B. Cunitz, V. Khokhlova, O. Sapozhnikov, and M. Bailey, Noninvasive acoustic manipulation of objects in a living body, Proc. Natl. Acad. Sci. 117, 16848 (2020).

[49] W.-C. Lo, C.-H. Fan, Y.-J. Ho, C.-W. Lin, and C.-K. Yeh, Tornado-inspired acoustic vortex tweezer for trapping and manippulating microbubbles, Proc. Natl. Acad. Sci. USA 118, e2023188118 (2021).

[50] N. Jiménez, V. Romero-García, L. M. García-Raffi, F. Camarena, and K. Staliunas, Sharp acoustic vortex focusing by fresnel-spiral zone plates, Appl. Phys. Lett. 112, 204101 (2018).

[51] M. Baudoin, J. Gerbedoen, B. M. O. N. Smagin, A. Riaud, and J.-L. Thomas, Folding a focalized acoustical vortex on a flat holographic transducer: Miniaturized selective acoustical tweezers, Sci. Adv. 5, eaav1967 (2019).

[52] M. Baudoin, J.-L. Thomas, R. Al Sahely, J.-C. Gerbedoen, Z. Gong, A. Sivery, O. B. Matar, N. Smagin, P. Favreau, and A. Vlandas, Spatially selective manipulation of cells with single-beam acoustical tweezers, Nat. Commun. 11, 4244 (2020).

[53] R. D. Muelas-Hurtado, J. L. Ealo, and K. Volke-Sepúlveda, Active-spiral fresnel zone plate with tunable focal length for airborne generation of focused acoustic vortices, Appl. Phys. Lett. 116, 114101 (2020).

[54] O. A. Sapozhnikov and M. R. Bailey, Radiation force of an arbitrary acoustic beam on an elastic sphere in a fluid, J. Acoust. Soc. Am. 133, 661 (2013).

[55] G. T. Silva, An expression for the radiation force exerted by an acoustic beam with arbitrary wavefront $(l)$, J. Acoust. Soc. Am. 130, 3541 (2011).

[56] Z. Gong and M. Baudoin, Equivalence between multipole expansion based and angular spectrum based threedimensional acoustic radiation force and torques formulas, J. Acoust. Soc. Am. 149, 3469 (2021).

[57] Z. Gong and M. Baudoin, Acoustic radiation torque on a particle in a fluid: An angular spectrum based compact expression, J. Acoust. Soc. Am. 11, 3131 (2020).

[58] P. J. Westervelt, The theory of steady forces caused by sound waves, J. Acoust. Soc. Am. 23, 312 (1951).

[59] P. J. Westervelt, Acoustic radiation pressure, J. Acoust. Soc. Am. 29, 26 (1957).

[60] P. Augustsson, J. T. Karlsen, H.-W. Su, H. Bruus, and J. Voldman, Iso-acoustic focusing of cells for size-insensitive acousto-mechanical phenotyping, Nat. Commun. 7, 11556 (2016).

[61] Z. Gong and M. Baudoin, Three-Dimensional Trapping and Assembly of Small Particles with Synchronized Spherical Acoustical Vortices, Phys. Rev. Appl. 14, 064002 (2020).

[62] P. Augustsson, R. Barnkob, S. T. Wereley, H. Bruus, and T. Laurell, Automated and temperature-controlled microPIV measurements enabling long-term-stable microchannel acoustophoresis characterization, Lab Chip 11, 4152 (2011).

[63] M. Settnes and H. Bruus, Forces acting on a small particle in an acoustical field in a viscous fluid, Phys. Rev. E 85, 016327 (2012).

[64] A. Riaud, M. Baudoin, J.-L. Thomas, and O. B. Matar, Cyclones and attractive streaming generated by acoustical vortices, Phys. Rev. E 90, 013008 (2014).

[65] P. Zhang, T. Li, J. Zhu, X. Zhu, S. Yang, Y. Wang, X. Yin, and $X$. Zhang, Generation of acoustic self-bending and bottle beams by phase engineering, Nat. Commun. 5, 4316 (2014).

[66] P. Brunet, M. Baudoin, O. B. Matar, and F. Zoueshtiagh, Droplet displacements and oscillations induced by ultrasonic surface acoustic waves: A quantitative study, Phys. Rev. E 81, 036315 (2010).

[67] D. Zhao, J.-L. Thomas, and R. Marchiano, Generation of spherical vortex beams to trap large particles for enhanced axial force, Ultrasonics 111, 106296 (2020). 